\title{
GAMMA-RAY CONTINUUM AND LINE OBSERVATIONS OF SN 1987A
}

\author{
D. M. Palmer, ${ }^{1}$ S. M. Schindler, W. R. CoOK, J. M. Grunsfeld, ${ }^{2}$ W. A. Heindl, \\ T. A. Prince, and E. C. Stone \\ Division of Physics, Mathematics, and Astronomy, California Institute of Technology, Mail Stop 220-47, Pasadena, CA 91125 \\ Received 1992 December 8; accepted 1993 January 27
}

\begin{abstract}
The Caltech $\gamma$-ray imaging telescope observed the hard X-ray and $\gamma$-ray emission from SN 1987A as it evolved between 1987 May and 1989 April, in a series of four balloon flights from Alice Springs, NT, Australia. Observations of the Crab Nebula and Pulsar provided in-flight data on pointing accuracy and instrument function for each flight. Results presented here include our measurements on 1987 November 18 (D268) and 1988 April 12 (D414) of both line emission at 847 and $1238 \mathrm{keV}$ from the decay of ${ }^{56} \mathrm{Co}$, and Comptonscattered continuum above $50 \mathrm{keV}$. Upper limits for both line and continuum emission were obtained on 1987 May 20 (D86) and 1989 April 4 (D771). For the D268 and D414 continuum measurements, our results are best-fit by a differential power law of the form $d N / d E=\kappa E^{-\gamma}\left(\mathrm{cm}^{-2} \mathrm{~s}^{-1} \mathrm{keV}^{-1}\right)$ for the energy range 50-800 $\mathrm{keV}$. Our corresponding line measurements were fit with Gaussian profiles, containing additional terms for the underlying continuum. We compare our flux measurements and upper limits to those from other experiments and to predictions of theoretical models.
\end{abstract}

Subject headings: gamma rays: observations - supernovae: individual (SN 1987A)

\section{INTRODUCTION}

SN 1987A, located in the Large Magellanic Cloud, provided a unique opportunity to study the evolution of the hard X-ray and $\gamma$-ray emission of a Type II supernova. For Type II supernovae, it was predicted that the strongest $\gamma$-ray line emission would occur more than a year after the explosion, resulting from the decay of ${ }^{56} \mathrm{Co}$ (synthesized as ${ }^{56} \mathrm{Ni}$ in the initial explosion) to ${ }^{56} \mathrm{Fe}$, with dominant line energies at 847 and $1238 \mathrm{keV}$ (e.g., Woosley, Axelrod, \& Weaver 1981). In contrast, the study of the continuum flux produced by Comptonization of the line-energy $\gamma$-rays in the ejecta had received less attention until SN 1987A demonstrated the importance of the continuum flux to an understanding of supernovae evolution. Detection of line flux at the ${ }^{56} \mathrm{Co}$ decay energies provides the most direct evidence of the synthesis of ${ }^{56} \mathrm{Ni}$ in the explosion, while the evolution of both line and continuum flux over time reflects the dynamics of the explosion and the subsequent mixing and motion of the ejecta.

Observations of SN 1987A at all wavelengths commenced almost immediately after its discovery in the visible region on 1987 February 24 (Shelton 1987). Using the Caltech GammaRay Imaging Payload (GRIP), a balloon-borne coded-aperture telescope, we made four flights from Alice Springs, NT, Australia (longitude: $133^{\circ} 52^{\prime} \mathrm{E}$; latitude: $-23^{\circ} 42^{\prime}$ ) to observe the evolution of the hard X-ray and $\gamma$-ray emission from SN 1987A between 1987 May and 1989 April. Our initial observations on 1987 May 20 (D86), ${ }^{3}$ yielded upper limits for the energy range $50 \mathrm{keV}$ to $10 \mathrm{MeV}$ (Cook et al. 1987). Other X-ray and $\gamma$-ray instruments observing the supernova during its first few months also obtained only upper limits, with the first detections occurring in 1987 July-August, 5 months after the initial observation at optical wavelengths (Dotani et al. 1987;

\footnotetext{
${ }^{1}$ Postal address: NASA/GSFC, Code 661, Greenbelt, MD 20771.

2 Postal address: NASA/JSFC, Code CB, Houston, TX 77058

${ }^{3}$ We have adopted the notation of designating our SN 1987A observations in terms of the number of days since the local detection, on 1987 February 23, of the neutrino burst from the supernova (e.g., 1987 May $20=$ day $86 \equiv$ D86).
}

Sunyaev et al. 1987; Englhauser et al. 1989; Matz et al. 1988). The subsequent two GRIP observations, on 1987 November 18 (D268) and 1988 April 12 (D414), measured both line and continuum flux (Cook et al. 1988a, b). Our results from the 1987 November 18 flight yielded the first imaging observation of the supernova at $\gamma$-ray energies, and confirmed that the bulk of the $\gamma$-ray emission came from SN 1987A, eliminating the nearby X-ray source, LMC X-1, as a significant source of emission (Cook et al. 1988c). At the time of our fourth observation on 1989 April 4 (D771), the hard X-ray and $\gamma$-ray emission had declined below the level detectable by our instrument.

We present here a description of our measurements and upper limits for both line emission at 847 and $1238 \mathrm{keV}$ from the decay of ${ }^{56} \mathrm{Co}$, and Compton-scattered continuum above $50 \mathrm{keV}$ for SN 1987A. These results incorporate a detailed re-analysis of our instrument response function based on laboratory measurements and modeling of photon attenuation in passive materials in the GRIP instrument (Palmer 1992). As discussed in $\S 4$, the wide energy range of our instrument, combined with its sensitivity to both line and continuum flux, allows a consistent intercomparison to be made between our measurements and a wide variety of other observations and models describing the evolution of SN 1987A. In particular, we compare our results to three models: Model 10HMM of Pinto \& Woosley (1988a), which was developed to explain the early emergence of X-ray and $\gamma$-ray flux from SN 1987A; Model 3 of Leising \& Share (1990, hereafter LS3); and the model described in Sunyaev et al. (1990, hereafter S90). Models LS3 and S90 were developed in response to observations of the later time evolution of the line and continuum emission, respectively.

\section{INSTRUMENT AND OBSERVATIONS}

The Caltech Gamma-Ray Imaging Payload is a balloonborne coded-aperture telescope, sensitive to radiation in the energy range from $30 \mathrm{keV}$ to $10 \mathrm{MeV}$ (Althouse et al. 1985). A rotating coded mask, composed of lead blocks mounted on an aluminum Hexcel support structure is located $2.5 \mathrm{~m}$ above the primary detector, a $5.1 \mathrm{~cm}$ thick by $41 \mathrm{~cm}$ diameter position- 


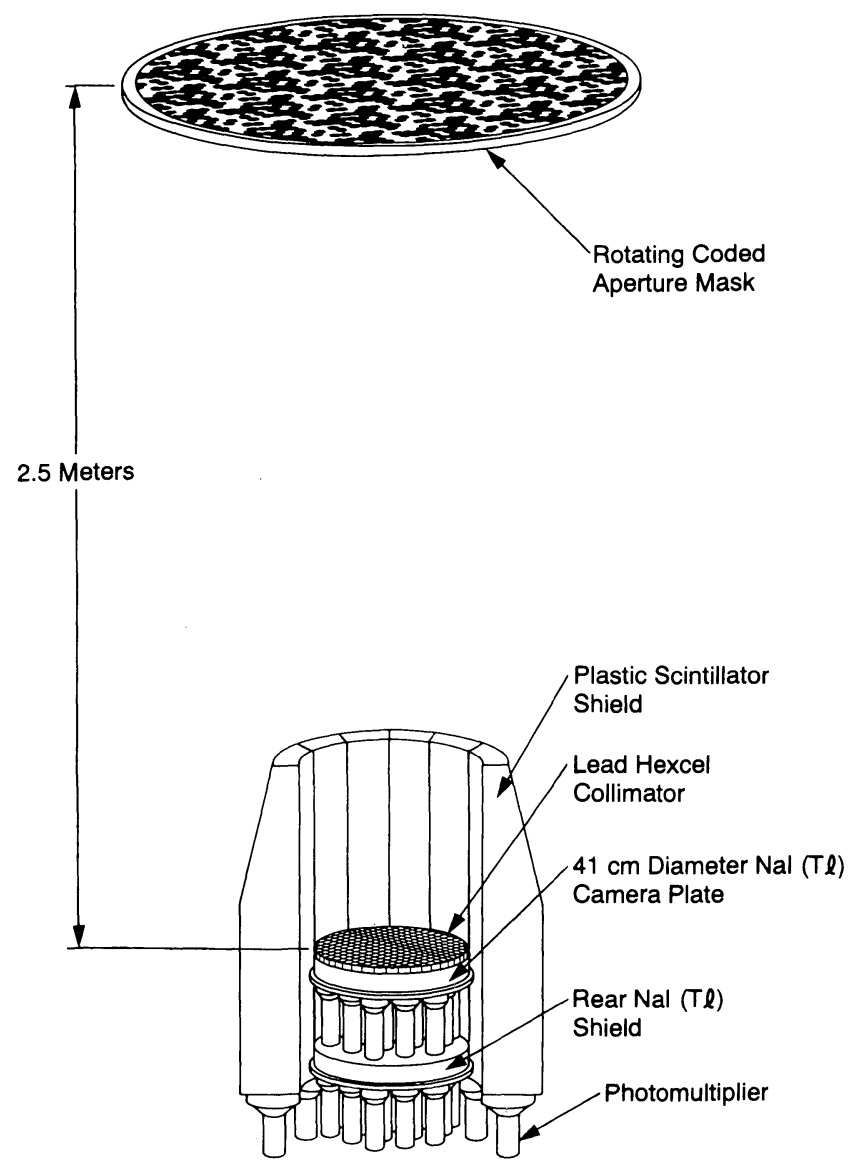

FIG. 1.-Schematic diagram of the GRIP instrument as configured for the D268, D414, and D771 observations. For the D86 observation the collimator was absent, and a different coded mask was used as described in the text.

sensitive $\mathrm{NaI}(\mathrm{Tl})$ scintillator (see Fig. 1). The mask pattern employed is a hexagonal-celled uniformly redundant array (HURA). Active shielding is provided by a $16 \mathrm{~cm}$ thick, cylindrical plastic scintillator surrounding the primary detector, and a second $41 \mathrm{~cm}$ diameter $\mathrm{NaI}(\mathrm{Tl})$ scintillator behind the primary detector. Self-shielding is also employed at energies below $300 \mathrm{keV}$, using a three-dimensional position algorithm which allows rear-incident $\gamma$-rays in the primary detector to be removed in post-flight processing. The energy resolution of the detector is measured to be $6.5 \% \mathrm{FWHM}$ at $1 \mathrm{MeV}$, and $16 \%$ FWHM at $100 \mathrm{keV}$. The flux sensitivity of the instrument was determined by a combination of laboratory measurement and Monte Carlo modeling. As discussed in $\S 3$, the instrument response was verified by in-flight measurements of the Crab Nebula and Pulsar, which are found to be in good agreement with other independent observations.

The telescope is mounted on a lightweight pointing platform, suspended from the balloon through a torque motor assembly which drives the system in azimuth. A redundant two-axis magnetometer system provides active feedback to the torque motor, allowing the platform to be driven to any bearing relative to magnetic north. Elevation motion of the telescope, relative to the pointing platform, is achieved through a ball-screw and stepping motor assembly coupled to the platform. Elevation reference is provided by precision inclinometers mounted on the platform, coupled with two independent shaft encoders on the elevation axis. Instrument pointing is controlled by an on-board microprocessor containing the detailed pointing plan. For the D414 and D771 observations, an image-intensified CCD star camera and an independent CCD Sun camera system were employed on the instrument to provide improved aspect information for post-flight pointing analysis for various sources of interest, in addition to SN 1987A. The star camera is sensitive down to seventh magnitude, but was not used during the observations of SN 1987A, which occurred during the daylight and twilight hours. The Sun camera was employed for single-axis tracking of the Sun, planets, and bright stars (to magnitude 2.5), while the telescope observed SN 1987A.

A "tagged" calibration source, consisting of ${ }^{241} \mathrm{Am}$ embedded in plastic scintillator and viewed by a single photomultiplier tube, is located appioximately $1 \mathrm{~m}$ above the coded mask, outside of the nominal field of view (FOV) of the telescope. The tagged events reaching the primary detector supply a lowbackground, monoenergetic signal coming from a known location in the FOV. This provides both a continuous measure of instrument operation, and a $60 \mathrm{keV}$ reference point for in-flight calibration of the energy scale. During the D771 observations, a malfunction in the ${ }^{241} \mathrm{Am}$ tagging system resulted in contamination of our observations at energies below $77 \mathrm{keV}$, although it did not prevent the calibration and function checks. Subsequent omission of data at these energies reduced the sensitivity of the D771 observation by less than 3\%.

The instrument was flown in two configurations for the supernova observations. The first configuration employed a coded mask having a repeating 79 cell unit pattern constructed from $2.5 \mathrm{~cm}$ (flat-to-flat) hexagonal lead blocks, yielding an angular resolution of 0.6 over a $20^{\circ}$ fully coded FOV. This configuration was used to observe the supernova in 1987 May (D86), and had been flown previously from Palestine Texas in 1986 October (Althouse et al. 1987). To enhance the instrument sensitivity for subsequent supernova observations, a second configuration was employed for the D268, D414, and D771 observations. For these observations, a coded mask having a repeating 31 cell pattern, constructed from $4.8 \mathrm{~cm}$ (flat-to-flat) hexagonal lead blocks, was employed. This resulted in an instrument angular resolution of 1.1 , with a $14^{\circ}$ fully coded FOV. In addition, a lead hexcell collimator having a nominal $10^{\circ}$ FWHM geometric FOV (for energies below $\sim 200 \mathrm{keV}$ ) was employed above the primary $\mathrm{NaI}(\mathrm{Tl})$ detector to reduce the diffuse background contribution at hard X-ray energies. These changes enhanced the flux sensitivity and efficiency of the instrument in three ways: First, the hexagonal lead blocks employed in the coded mask pattern were thicker in the second configuration $(2.8$ vs. $1.9 \mathrm{~cm})$, making the mask more effective at the 847 and $1238 \mathrm{keV}$ line energies expected from the supernova. Second, the larger mask cell size resulted in an improved flux sensitivity for the instrument, at the expense of angular resolution (Finger 1987). Finally, the lead hexcell collimator significantly improved our on-axis flux sensitivity at energies below $\sim 200 \mathrm{keV}$, though with an associated reduction in sensitivity near the edge of the instrument's FOV. For the supernova observations, fine angular resolution and wide FOV were not primary requirements due to the well-defined source position.

Table 1 summarizes the observational details of the four GRIP observations of SN 1987A. Due to changes in the instrument configuration as described above, coupled with differences in both the atmospheric overburden and length of observation for each flight, the sensitivity of the instrument 
TABLE 1

SUMMARY OF GRIP OBSERVATIONS OF SN 1987A

\begin{tabular}{|c|c|c|c|}
\hline $\begin{array}{l}\text { Observation } \\
\text { (Day number) }\end{array}$ & Date & $\begin{array}{l}\text { SN 1987A } \\
\text { Livetime } \\
\text { (hr) }\end{array}$ & $\begin{array}{l}\text { Continuum Sensitivity } \\
\left(10^{-5} \mathrm{~cm}^{-2} \mathrm{~s}^{-1} \mathrm{keV}^{-1}\right)\end{array}$ \\
\hline D86 & 1987 May 20 & 5.4 & 1.8 \\
\hline D268 .... & 1987 Nov 18 & 2.3 & 2.1 \\
\hline D414 $\ldots \ldots \ldots \ldots \ldots$ & 1988 Apr 12 & 11.1 & 1.2 \\
\hline D771 ............. & 1989 Apr 4 & 3.3 & 1.9 \\
\hline
\end{tabular}

a Continuum sensitivity is the flux at $100 \mathrm{keV}$ that would result in a $3 \sigma$ detection of the supernova, assuming an $E^{-1}$ power-law spectrum between 50 and $800 \mathrm{keV}$.

varied for each of the observations. These factors are reflected in the sensitivity levels shown in Table 1 . As discussed in $\S 3$, a typical pointing sequence for each flight consisted of a series of nominal 1 hour supernova pointings, interspersed with relatively short pointings ( $\sim 20-30$ minutes) at the Crab Nebula and Pulsar. The Crab pointings provided checks on both pointing accuracy and instrument calibration for the associated supernova observations. A total of $17 \mathrm{Crab}$ pointings were made during the four flights done to observe SN 1987A.

\section{ANALYSIS TECHNIQUES}

Analysis of data from a coded-aperture instrument produces an X-ray or $\gamma$-ray image of a potential source region on the sky. The image construction procedure assigns each resolution element, $\boldsymbol{x}$, on the image a value, $I(\boldsymbol{x})$, the so-called "image value." This corresponds to the telescope response to flux from the region centered on the corresponding point on the sky. ${ }^{4}$ The size of the region corresponds to the angular resolution of the instrument. Imaging with a rotating HURA uses each point on the detector to measure both the background level and the source strength from each point in the sky, alternating continuously between background and source as the mask rotates. This technique removes both spatial and temporal variations in the detector background. For the GRIP instrument, both the analysis procedure and algorithm used to generate the image are described in detail in Cook et al. (1984) and Finger (1987).

Converting from a set of source images made over a given energy range, to a source flux in the same energy range, requires consideration of several energy-dependent factors. These include the imaging efficiency of the telescope, the lineof-sight opacity of both the instrument material and atmosphere, and for the case of the GRIP instrument, the finite energy resolution of the $\mathrm{NaI}(\mathrm{Tl})$ detector. It is anticipated that the source flux will be energy-dependent, such that a single derived value will not be representative over the entire energy range of interest. Therefore, an energy-dependent image analysis is employed, using the method described by Heindl et al. (1993). In this approach a trial spectrum, or "template," $T(E)$, with unit intensity, is initially chosen to represent the shape of the source spectrum. The template is convolved with the instrument response function of the detector, $R(E, x)$, to calculate the image value corresponding to a source having a spectrum equal to the unit-intensity template. That is

$$
\left\langle I_{i}(\boldsymbol{x})\right\rangle_{T}=\int R_{i}(E, \boldsymbol{x}) T(E) d E,
$$

\footnotetext{
${ }^{4}$ The image value can be compared to the background-subtracted countrate-spectrum from a nonimaging instrument, and includes those factors associated with the imaging properties of the detector.
}

where the subscript $i$ specifies an individual image, which include the image-associated instrumental and observational parameters, and $E$ denotes the photon energy. For the background-dominated case, corresponding to the measurements described here for SN 1987A, the variance associated with the image value is independent of the source flux, and is given by

$$
\begin{aligned}
\left(\sigma_{i}\right)^{2} & =\left\langle\left[I_{i}(x)\right]^{2}\right\rangle_{T} \\
& =\epsilon(E) N_{i},
\end{aligned}
$$

where $N_{i}$ is the total number of photons used in the image reconstruction, and $\epsilon(E)$ is the so-called "imaging factor" (Finger 1987), a number of order unity reflecting the effect of the finite position resolution of the GRIP detector on the statistics of the measurement.

The calculated response to the template spectrum is compared to the measured image value, $I_{i}(\boldsymbol{x})$, the ratio of which yields an estimator of the measured source flux to that obtained from the template, or the flux normalization, $\kappa_{i}$ :

$$
\kappa_{i}=\frac{I_{i}(\boldsymbol{x})}{\left\langle I_{i}(\boldsymbol{x})\right\rangle_{T}},
$$

which provides a measure of the source flux, $f(E, x)$ at $\boldsymbol{x}$, where

$$
f_{i}(E, \boldsymbol{x})=\kappa_{i} T(E) \text {. }
$$

The estimators $\kappa_{i}$, calculated for images made over different energy ranges and/or time periods, are linearly combined to achieve greater statistical significance than is available from any individual image:

$$
\kappa=\left(\sigma_{\kappa}\right)^{2} \sum_{i} \frac{I_{i}(\boldsymbol{x})\left\langle I_{i}(\boldsymbol{x})\right\rangle_{T}}{\left(\sigma_{i}\right)^{2}},
$$

with

$$
\left(\sigma_{\kappa}\right)^{2}=\left[\sum_{i} \frac{\left(\left\langle I_{i}(x)\right\rangle_{T}\right)^{2}}{\left(\sigma_{i}\right)^{2}}\right]^{-1} .
$$

The consistency of the individual estimators provides a measure of the validity of the selected template, which is then iteratively adjusted to obtain the best fit. Although the resulting flux is template-dependent (Fenimore et al. 1983), this dependence is reduced by stating the flux at a nominal energy, chosen using the "bow tie" method (Van Allen et al. 1974). In this approach, the spectra derived from the same data for different templates are plotted. For the range of spectral parameters employed in the analysis described here, the spectra tend to cross each other near a given point (the "knot" of the "bow tie"), which defines a single energy and flux which adequately describes the spectra.

For our analysis of SN 1987A between 50 and $800 \mathrm{keV}$, we employ a power-law template of the form $d N / d E \propto E^{-\gamma}$. The use of a power law approximates the results of a wide variety of Monte Carlo models (e.g., 10HMM and S90, referenced in $\S 1$, and others referenced in $\S 5$ ). Above $800 \mathrm{keV}$, the energy dependence of the instrument response is reduced, and the spectrum is also more model-dependent. For this case we employed a flat template with constant $d N / d E$, corresponding to a $\gamma=0$ power law. In the analysis, the data were divided into narrow (less than $10 \mathrm{keV}$ at energies below a few hundred $\mathrm{keV})$ measured-energy ranges over short $(\sim 1 \mathrm{hr})$ time periods to produce a series of statistically independent images during each observation. These images are not individually statistically significant, but are summed using weights derived 


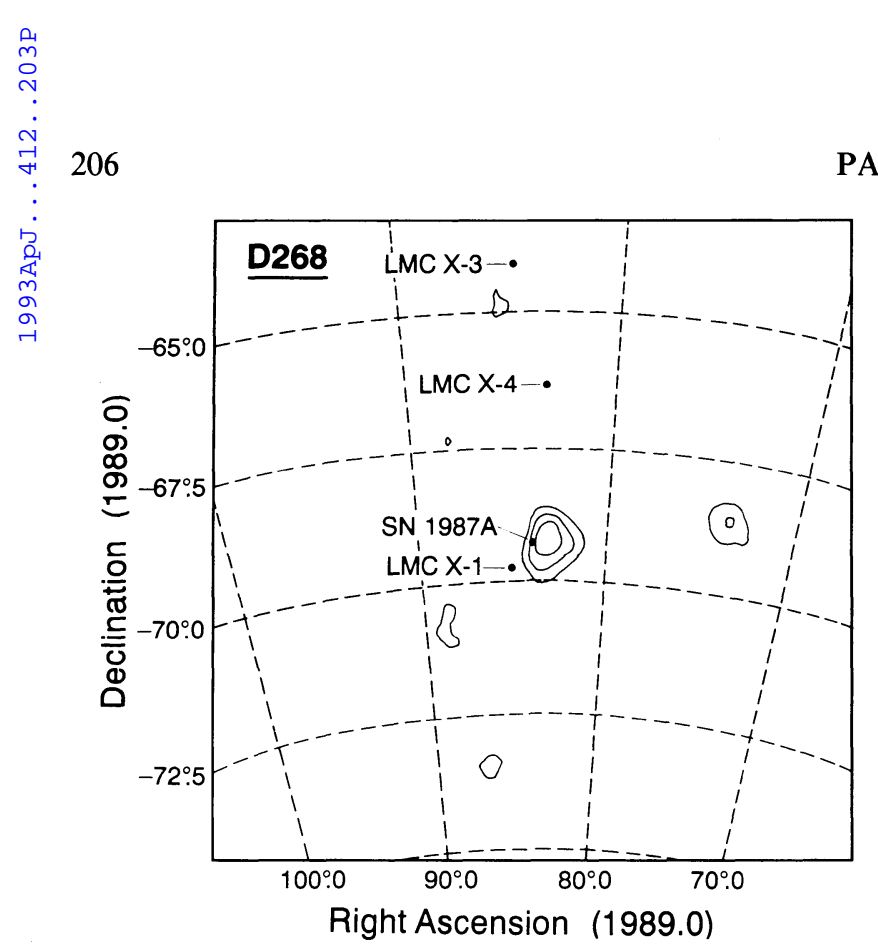

FiG. $2 a$

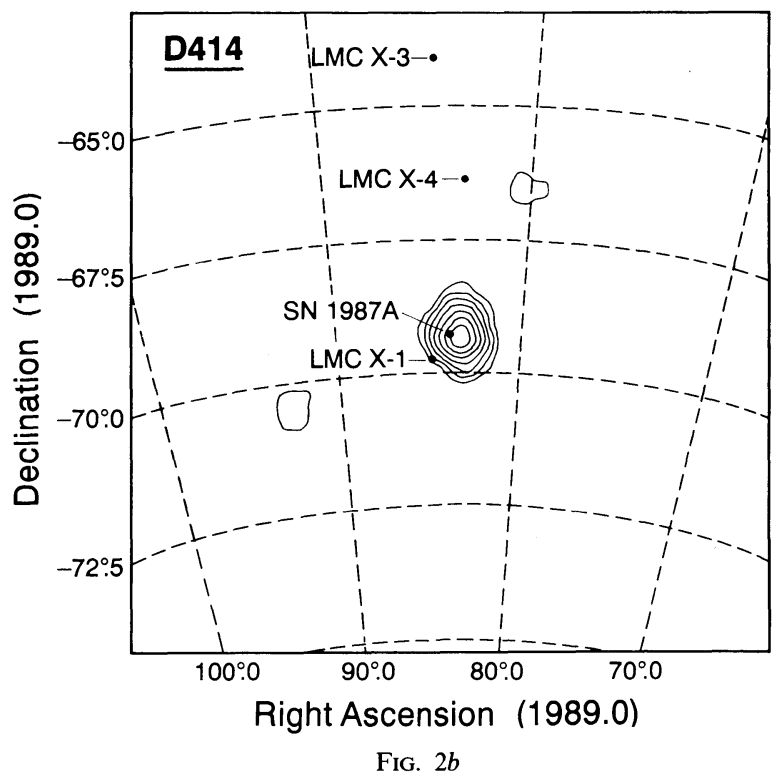

FIG. 2.-Images of the SN 1987A region for the D268 (Fig. $2 a$ ) and D414 (Fig. $2 b$ ) observations from 50 to $1600 \mathrm{keV}$, covering a nominal $14^{\circ}$ field of view. Right

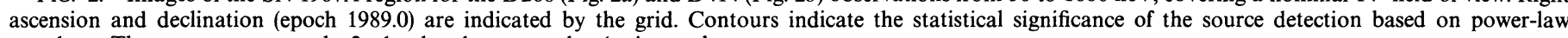
templates. The contours start at the $2 \sigma$ level and are spaced at $1 \sigma$ intervals.

from the template to yield a "composite" image which is statistically optimal for a source with a spectrum matching the template (Palmer 1992). The D268 and D414 observations resulted in the images shown in Figure 2. As discussed further in $\S 4$, these images have statistically significant peaks within $\sim 0.3$ of the location of SN 1987A, with significances of 4.9 and $8.8 \sigma$, respectively. Physically reasonable templates for the D68 and D771 observations did not reveal any significant $(\geq 3 \sigma)$ peaks near the location of SN 1987A. These trial templates included power-laws with $\gamma$ in the range 0 through 5 , thermal bremsstrahlung spectra for temperatures from $10 \mathrm{keV}$ to 1 $\mathrm{MeV}$, and, for the D771 observation, templates based on Monte Carlo calculations (e.g., Grebenev \& Sunyaev 1989) that are dominated by the line and continuum flux from ${ }^{57} \mathrm{Co}$, at and below $122 \mathrm{keV}$.

Because an imaging instrument produces a separate measurement for each resolution element in the sky, the precise location of the source on the image is determined before a spectrum is calculated. If both the instrument pointing and the location of the source on the sky are known exactly, the image value at the nominal source location provides an unbiased estimator of the flux. When there is an evident image peak due to the source, and the systematic pointing error exceeds the statistical position error, the source flux is obtained from the maximum of the source peak. This approach introduces a small positive bias in the measured flux, proportional to the variance of the measured position (Finger 1987). For the D268 and D414 observations, we used the peak of the composite images shown in Figure 2 to determine the source flux. While this point depends, in theory, on the template used, we find that for the D268 and D414 observations, for spectral indices in the range $0 \leq \gamma \leq 3$, the resulting shift is less than the 0.1 pixel size used in our image construction. For the image points obtained from the optimized composite images, the positive bias in the SN 1987A spectral measurement is estimated to be $4.2 \%$ for D268 and $1.3 \%$ for D414, well below the statistical errors of the measurements.

For source observations below the detection threshold of the instrument (e.g., the D86 and D771 observations of SN 1987A, which do not yield a significant image peak), upper limits are obtained for the point on the image corresponding to the best estimate of the instrument pointing direction. A more conservative upper limit is obtained by using the image value at the highest point on the composite image, consistent with the estimated maximum pointing error of the telescope. For the upper limits on the $50-800 \mathrm{keV}$ continuum flux for D86 and D771 given in Table 2 , the choice of the highest value consistent with a $1^{\circ}$ maximum pointing error, raises the upper limits by $15 \%$ and $3 \%$, respectively. In both cases, the highest point is consistent with statistical fluctuations for a field containing no sources. As discussed below, the use of a $1^{\circ}$ maximum pointing error for the D86 and D771 observations is conservative, given the measured residual pointing errors of 0.3 and 0.2 obtained for the D286 and D414 observations, respectively.

For each of our four supernova observations, the instrument pointing direction alternated between SN 1987A (observed for $\sim 1 \mathrm{hr}$ periods) and the Crab Nebula and Pulsar (observed for $\sim 20-30$ minute periods). The Crab provides a source with a known location and approximately constant intensity, allowing us to determine pointing offsets and verify instrument response. Analysis of the $\mathrm{Crab}$ data after post-flight pointing

TABLE 2

Measured Spectral Parameters for SN 1987A ContinuUm FLUX, INCLUDING UPPER Limits AT THE 95\% CONFIDENCE LEVEL

\begin{tabular}{|c|c|c|}
\hline Observation & $\begin{array}{c}\text { Spectral Index } \\
\gamma\end{array}$ & $\begin{array}{c}\kappa_{100} \\
\text { Flux at } 100 \mathrm{keV} \\
\left(10^{-5} \mathrm{~cm}^{-2} \mathrm{~s}^{-1} \mathrm{keV}^{-1}\right)\end{array}$ \\
\hline$\ldots \ldots$ & 1 & $<2.0$ \\
\hline D268 $\ldots \ldots \ldots \ldots \ldots \ldots \ldots \ldots$ & $1.3 \pm 0.4$ & $3.4 \pm 1.1$ \\
\hline D414 $\ldots \ldots \ldots \ldots \ldots \ldots \ldots$ & $0.97 \pm 0.17$ & $3.3 \pm 0.6$ \\
\hline D771 $\ldots \ldots \ldots \ldots \ldots \ldots \ldots$ & 1 & $<1.6$ \\
\hline
\end{tabular}

NoTE.-To calculate upper limits for D86 and D771, the spectral index was assumed to be 1 . 
corrections indicated that the residual pointing error had an rms deviation, averaged over all four observations, of 0.54 , with a range of less than $1^{\circ}$ over each individual flight. This error was primarily in the azimuthal direction, and varied systematically over the course of each flight. To analyze the SN 1987A data for the D86 and D268 flights, the systematic offsets (azimuth and elevation) found for the individual Crab pointings were interpolated and applied to the analysis of the supernova pointings. Following this procedure, the residual image peak displacement found for SN 1987A for the D268 observation was 0.3 , of which 0.09 is the statistical contribution resulting from photon number statistics of the image. The residual displacement is at the level corresponding to the design envelope for the instrument, and implies nominal pointing performance. For the D414 and D771 flights, the Sun camera provided aspect information which constrained one degree of freedom (the angular distance between the Sun and the telescope pointing direction). Because the elevation offset was found to be more stable over time than the azimuthal offset, the elevation offset for the D414 and D771 observations was obtained from interpolation of the Crab data, while the azimuthal offset incorporated the Sun camera data. Offsets derived from the Sun camera data were similar in magnitude and systematic behavior to the Crab-derived offsets. The residual peak displacement for the D414 observation was found to be 0.2 , of which 0.05 is the statistical contribution. As discussed in $\S 4$, the residual displacements tend to be in a direction resulting from a systematic azimuthal error in telescope pointing.

To verify our analysis technique and calibration of the instrument response for each of the four supernova observations, the data from each observation of the Crab Nebula and Pulsar were analyzed using the same imaging and analysis techniques as were applied to the SN 1987A data. Seven of the 17 Crab pointings were made during the D414 flight, totaling 2.0 livetime hours. Using the D414 data as an example, images were generated for each of the seven Crab pointings. These data were fit to a power-law spectrum of the form $d N / d E=$ $\kappa_{100}(E / 100 \mathrm{keV})^{-\gamma}$ between 50 and $800 \mathrm{keV}$, resulting in a best fit (reduced $\chi^{2}=0.84$; probability of exceeding $\chi^{2}:>99.7 \%$ ) with $\gamma=2.23 \pm 0.06$, and a flux at $100 \mathrm{keV}$ of $\kappa_{100}=(55.2$ $\pm 1.3) \times 10^{-5}\left(\mathrm{~cm}^{2} \mathrm{~s} \mathrm{keV}\right)^{-1}$. This spectrum was used as a template to calculate the differential flux of the Crab.

The results of the analysis of the D414 Crab observations are shown in Figure 3. For comparison, the solid curve shows the results of the $H E A O \mathrm{~A}-4$ observations of the Crab made during the years 1977 and 1978 (Jung 1989). Our measurements, and those from $H E A O \mathrm{~A}-4$, are consistent in overall normalization and slope at the $10 \%$ level, providing an additional degree of confidence in our results. For the energy range analyzed, these results provide a measure of the apparent stability of the Crab emission for the time interval corresponding to the approximate decade between the observations.

\section{RESULTS}

Figures $2 a$ and $2 b$ are images of the sky region containing the supernova obtained from the D268 and D414 observations, respectively. These are composite images, produced using power-law continuum templates between 50 and 1600 $\mathrm{keV}$, and incorporate post-flight pointing corrections. Flux is detected at peak significance levels of 4.9 and $8.8 \sigma$, respectively, for the supernova. A similar analysis for D86 and D771

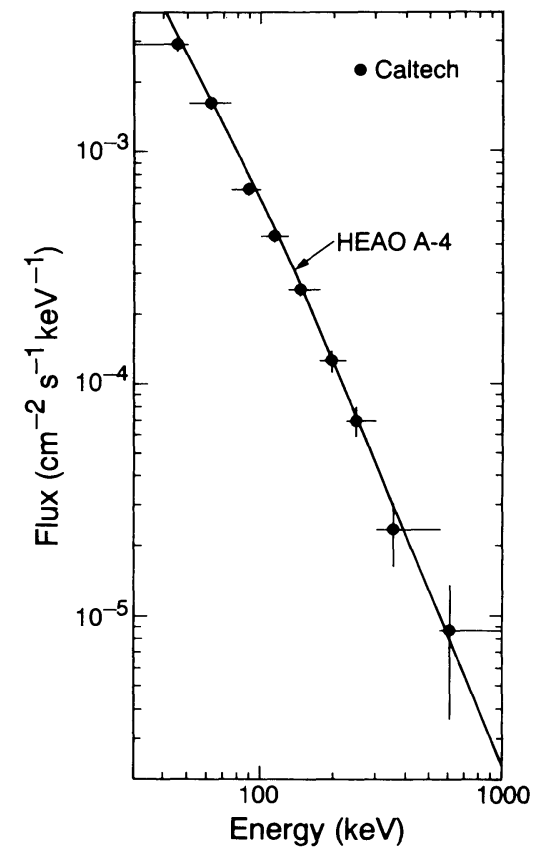

FIG. 3.-Comparison of the Caltech measurements of the Crab Nebula and Pulsar from the D414 flight with the HEAO A-4 spectrum for 1977-1978(Jung 1989). The analysis technique is identical to that used for the SN 1987A observations.

shows no evidence of excess flux from the region of the sky containing the supernova.

The displacements of the image peaks from the expected position of the supernova, in degrees of arc are $\Delta$ R.A. $=-0.3$, $\Delta$ decl. $=0.0$ for D268; and $\Delta$ R.A. $=-0.2, \Delta$ decl. $=-0.1$ for D414. As discussed in $\S 3$, these displacements result primarily from systematic variations in the instrument pointing direction. The statistical component associated with these displacements are 0.09 and 0.05 for D268 and D414, respectively, in each axis. The displacements are primarily along the telescope azimuth direction (which, on average, approximate deviations in R.A.), while in the telescope elevation direction ( $\sim$ declination), the centroid of the image peak is consistent, within statistical errors, with the position of SN 1987A. A more detailed analysis shows that, assuming all residual pointing errors are in the azimuthal direction, the location of the image peak is consistent with the known location of SN 1987A, and not with the location of the hard X-ray source LMC X-1 (Palmer 1992). Therefore, we use the peak positions for analysis of the D268 and D414 observations reported here.

The data from each supernova observation were fit to a differential power law spectrum of the form $d N / d E=\kappa(E / 100$ $\mathrm{keV})^{-\gamma}$ for the energy range $50-800 \mathrm{keV}$. For the D268 and D414 observations, both the spectral index $(\gamma)$ and the normalization $(\kappa)$ were allowed to vary, with $\kappa$ and $\gamma$ chosen to minimize $\chi^{2}$. The uncertainties for $\kappa$ and $\gamma$ given here are the deviations that result in a unity increase in $\chi^{2}$, when minimized with respect to the other parameter. This uncertainty corresponds to a $68 \%$ confidence level for each parameter individually (Lampton, Margon, \& Bowyer 1976; Avni 1976; Heindl et al. 1993). For the D86 and D771 observations, the spectral index was fixed at $\gamma=1$ so that upper limits could be calculated. Unless otherwise specified, all upper limits are at the $95 \%$ confidence level. This level is defined, for a quantity such as flux which is physically constrained to be non-negative, such 
TABLE 3

Measured ContinuUm FluX For SN 1987A

\begin{tabular}{|c|c|c|c|c|c|c|}
\hline \multicolumn{3}{|c|}{$\begin{array}{l}\text { ENERGY BIN } \\
(\mathrm{keV})\end{array}$} & \multicolumn{4}{|c|}{$\begin{array}{l}\text { FLUX AT NOMINAL ENERGY } \\
\left(10^{-5} \mathrm{~cm}^{-2} \mathrm{~s}^{-1} \mathrm{keV}^{-1}\right)\end{array}$} \\
\hline Minimum & Nominal & Maximum & D86 & D268 & D414 & D771 \\
\hline 50 & 75 & 100 & $<9.11$ & $6.04 \pm 2.47$ & $3.02 \pm 1.48$ & $<6.05^{\mathrm{a}}$ \\
\hline 100 & 135 & 175 & $<2.99$ & $2.23 \pm 0.86$ & $2.78 \pm 0.48$ & $<1.89$ \\
\hline 175 & 225 & 300 & $<1.29$ & $1.04 \pm 0.61$ & $1.64 \pm 0.35$ & $<0.69$ \\
\hline 300 & 425 & 550 & $<0.61$ & $0.35 \pm 0.40$ & $0.74 \pm 0.23$ & $<0.84$ \\
\hline 550 & 650 & 811 & $<0.49$ & $0.55 \pm 0.38$ & $0.40 \pm 0.20$ & $<1.05$ \\
\hline 811 & 847 & 883 & $<0.89$ & $1.53 \pm 0.75$ & $1.24 \pm 0.38$ & $<1.43$ \\
\hline 883 & 1050 & 1190 & $<0.40$ & $0.60 \pm 0.37$ & $0.06 \pm 0.19$ & $<0.76$ \\
\hline 1190 & 1238 & 1286 & $<0.71$ & $1.15 \pm 0.65$ & $0.08 \pm 0.32$ & $<0.64$ \\
\hline 1286 & 1450 & 1600 & $<0.70$ & $0.34 \pm 0.42$ & $0.13 \pm 0.21$ & $<0.71$ \\
\hline 1600 & 2500 & 3500 & $<0.09$ & $0.03 \pm 0.10$ & $0.06 \pm 0.05$ & $<0.20$ \\
\hline 3500 & 5000 & 10000 & $<0.02$ & $-0.05 \pm 0.05$ & $0.00 \pm 0.02$ & $<0.08$ \\
\hline
\end{tabular}

a Low-energy range for D771 is $77-100 \mathrm{keV}$. See $\S 2$.

that if a fraction $F$ of the normal distribution defined by the measurement is greater than $0,95 \% \times F$ of the distribution is between 0 and the $95 \%$ confidence level upper limit (Palmer 1992). These results are shown in Table 2 for the four supernova observations.

Our measurements and upper limits for the $\gamma$-ray continuum flux from SN 1987A are shown in Table 3. For each observation, the template is a power-law spectrum $(\gamma=1.0$ for D86 and D771; $\gamma=1.3$ for D268; $\gamma=0.97$ for D414) between 50 and $800 \mathrm{keV}$, and a flat spectrum $(\gamma=0)$ above $800 \mathrm{keV}$. The results in Table 3 are the differential photon number flux corresponding to the nominal energy of each bin. As mentioned in $\S 3$, this energy was chosen by the "bow tie" method to reduce the sensitivity of the flux values to variations in $\gamma$, noting that with the exception of the highest energy bin in Table 3 , the nominal energy is within $\sim 6 \%$ of the mean bin energy. Using the nominal energy, we find that a unity change in $\gamma$ results in a change in each derived flux value of less than $0.25 \sigma$, and in each upper limit of less than $25 \%$. The flux measurements for each energy bin depend only on those photons with measured energy inside the bin-cross-contributions due to the finite energy resolution of the detector are included in the forward energy convolution. With the exception of the narrow bins at the 847 and $1238 \mathrm{keV}$ line energies, all energy bins are at least $8 \sigma$ wide with respect to the measured instrument energy resolution, thus the flux contribution due to photons with energies outside the bin is small ( $\lesssim 10 \%$ of the calculated flux).

Our measurements for the D268 and D414 observations are shown in Figures $4 a$ and $4 b$ as a differential energy flux (differential photon number flux times energy). Also shown are the fitted power-law spectra from Table 2, and the results of the 10HMM and S90 Monte Carlo models.

As seen in Figures $4 a$ and $4 b$, our measured continuum flux between 50 and $800 \mathrm{keV}$ is a somewhat better fit to the results of the S90 model than to those of the 10HMM model. A detailed comparison indicates that our measurements are typically in agreement with the $\mathrm{S} 90$ predictions at the $10 \%$ level, and are approximately $30 \%$ below those of the $10 \mathrm{HMM}$
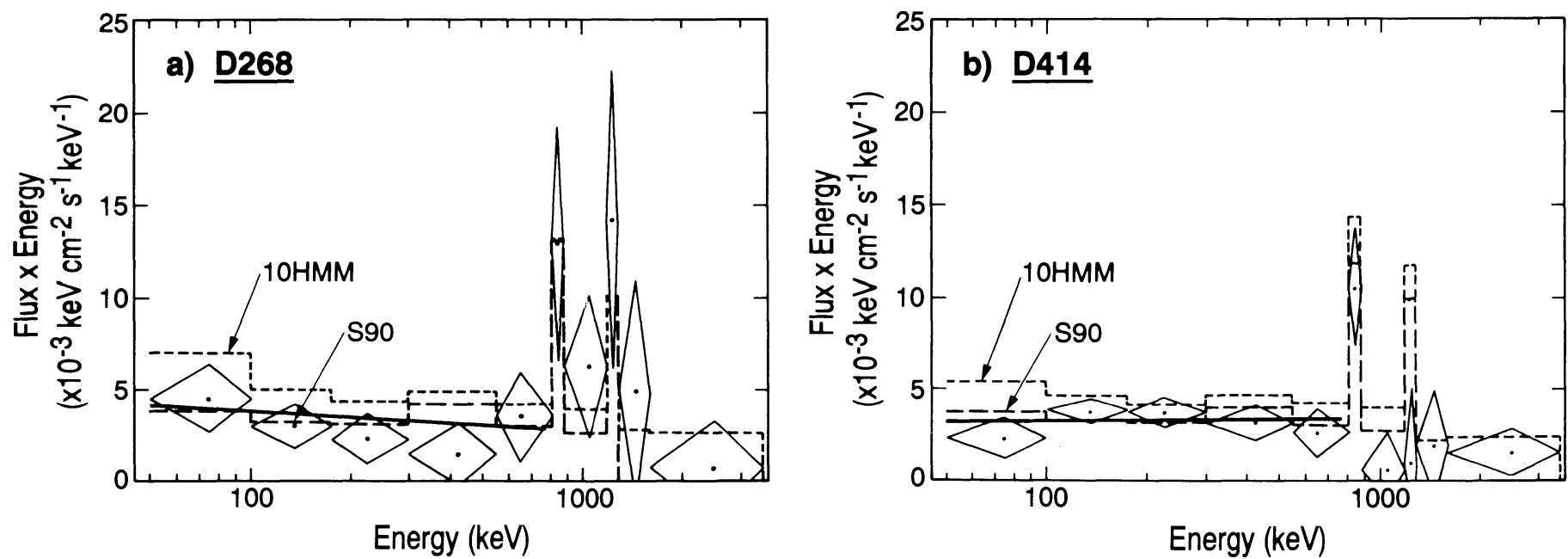

FIG. 4.-Differential energy flux spectra for the D268 (Fig. 4a) and D414 (Fig. 4b) observations. Our measured values are shown as diamonds, with the width

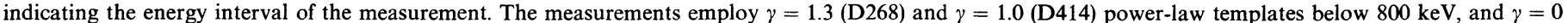

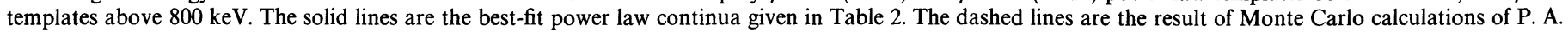

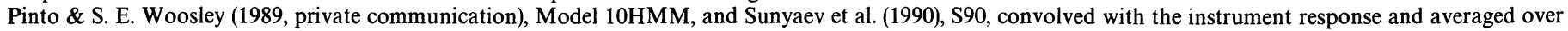
the energy intervals. 

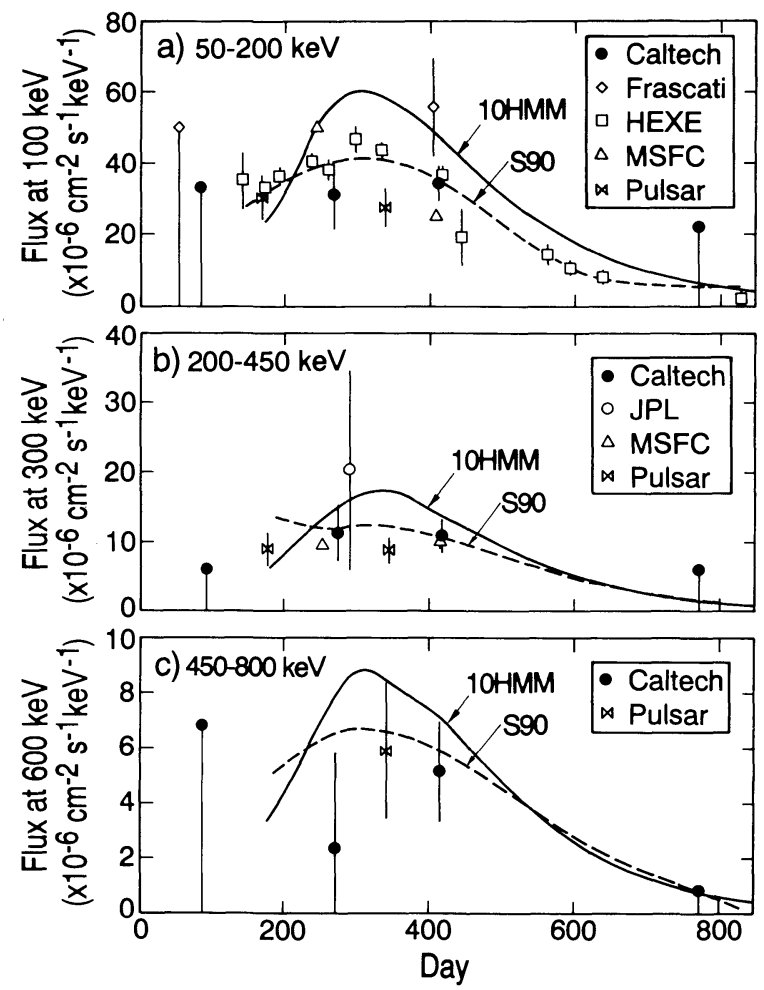

FIG. 5.-Comparison of GRIP continuum flux measurements with other observations of SN 1987A, and with Monte Carlo models. The energy ranges are: (a) $50-200 \mathrm{keV}$, (b) $200-450 \mathrm{keV}$, and (c) $450-800 \mathrm{keV}$, with fluxes normalized for a $\gamma=1$ template in each energy range and quoted at energies of: $(a)$ $100,(b) 300$, and (c) $600 \mathrm{keV}$. Upper limits are at the $95 \%$ confidence level for the D86 and D771 measurements, and at the $3 \sigma$ level for the D55 Frascatti measurements. The solid and dashed lines are the predicted continuum flux from P. A. Pinto \& S. E. Woosley (1989, private communication) Model 10HMM, and Sunyaev et al. (1990), Model S90, respectively. The data references are: Frascatti (Sood et al. 1988; Ubertini et al. 1989), HEXE and Pulsar (Sunyaev et al. 1990), MSFC (Fishman et al. 1990), and JPL (Mahoney et al. 1988).

model. The better agreement found with the $S 90$ model is due, in part, to the inclusion of additional observational data in S90 that was not available at the time $10 \mathrm{HMM}$ was developed.

Other instruments, in addition to GRIP, measured the line and continuum flux of SN 1987A. Figure 5 summarizes the continuum measurements and upper limits between 50 and 800 $\mathrm{keV}$ (references given in the figure caption). To simplify comparison, the measurements have been divided into three broad energy bands, with the data in each energy band normalized to specific energies by a fit to power-law templates with $\gamma=1$. In the 50-200 keV region, where detailed comparisons are possible, the measurements are in general agreement. Over the entire energy region of $50-800 \mathrm{keV}$, the observations are typically below the predictions of the 10HMM model, and in reasonable agreement with the $S 90$ model (see $\S 5$ ).

For the results shown in Table 3 and Figures $4 a$ and $4 b$, the nominal energy and width of the bins containing the 847 and $1238 \mathrm{keV}$ lines were chosen to maximize the sensitivity to excess flux due to line emission, based on our measured instrument energy resolution of $7 \%$ and $6.5 \% \mathrm{FWHM}$ at the two line energies, respectively. These bins include contributions from the underlying continuum and contain approximately $85 \%$ of the total line flux. To separate these components, the flux as a function of measured energy between 600 and $1750 \mathrm{keV}$ was analyzed in narrow bins having widths less than 0.35 of the FWHM instrument energy resolution. These data were fit with Gaussian line profiles, having widths based on the measured energy resolution of our instrument at the two line energies. For the D268 and D414 observations, an $F$-test for $\chi^{2}$ was applied to determine the validity of adding an additional term for the underlying continuum component (Bevington 1969). This analysis indicated (at better than the $95 \%$ confidence level) that the addition of a continuum component for both the D268 and D414 observations significantly reduced the $\chi^{2}$ of the fit, justifying the addition of the term. We have represented the continuum term by a $\gamma=1$ power-law, stepped at each line so that the continuum flux $f(E)$ is given by

$$
f(E)=\kappa_{800} \phi(E)(E / 800 \mathrm{keV})^{-1},
$$

where

$$
\phi(E)=\left\{\begin{array}{rr}
1 & E<847 \mathrm{keV} \\
0.57 & 847 \mathrm{keV}<E<1238 \mathrm{keV} \\
0.28 & 1238 \mathrm{keV}<E<1750 \mathrm{keV}
\end{array}\right.
$$

is proportional to the sum of all theoretical line strengths above $E$. The known ${ }^{56} \mathrm{Co}$ lines between the 847 and $1238 \mathrm{keV}$ lines have small branching ratios, and are ignored for simplicity. The effect of the ${ }^{56} \mathrm{Co}$ lines above $1238 \mathrm{keV}$ is included in the continuum model by treating this emission as resulting from a single line above $1750 \mathrm{keV}$. This representation of the continuum flux approximates the spectrum found in the Monte Carlo models discussed in $\S 5$. For each observation, a $\chi^{2}$ fit was made to two models: In Model A the line strengths are assumed independent of each other, while in Model B, the line strengths are constrained to the natural ${ }^{56} \mathrm{Co}$ branching ratio of 1:0.68. The results for Model A give our best estimate for the individual line strengths from SN 1987A. If the ${ }^{56} \mathrm{Co}$ line-energy photons escape from SN 1987A while retaining the natural branching ratio, Model $B$ gives the best estimate of the strength of the line complex. An F-test of the Model A and B fits indicates that our measurements are consistent with the natural branching ratio, and do not require an additional free parameter for the line flux ratio. The natural branching ratio is also consistent with measurements made by the GammaRay Spectrometer (GRS) aboard the Solar Maximum Mission (SMM), which gives a line flux ratio of $f(1238)$ / $f(847)=0.68 \pm 0.12$, averaged over the period 1987 August to 1988 May (Leising \& Share 1990). The results for both models are shown in Table 4.

Figure 6 compares our Model B line measurements with the results obtained from other instruments. For each of these observations, the line measurements were combined to give an $847 \mathrm{keV}$ equivalent flux. This was done by converting the 1238 $\mathrm{keV}$ line flux to an equivalent $847 \mathrm{keV}$ flux using the natural branching ratio, and then averaging the line flux. In those cases where a measurement was reported for one line, and an upper limit or no result reported for the other, the measured line flux is used. The smooth curves shown in Figure 6 are the line flux predictions of 10HMM (Pinto \& Woosley 1989), and Model 3 of Leising \& Share (1990), denoted LS3. The S90 result (Sunyaev et al. 1990) falls between or near the 10HMM and LS3 models, and is omitted for clarity. A comparison of these measurements shows that, within statistics, our results are in agreement with those made by other instruments at similar times. It is seen that these measurements do not strongly differentiate between the two models shown, based solely on line flux predictions. 
TABLE 4

MEASURED FluX FOR THE 847 AND $1238 \mathrm{keV}$ LiNeS FROM SN 1987A

\begin{tabular}{|c|c|c|c|c|}
\hline Observation & Model & $\begin{array}{l}847 \mathrm{keV} \text { Line Flux } \\
\left(10^{-4} \mathrm{~cm}^{-2} \mathrm{~s}^{-1}\right)\end{array}$ & $\begin{array}{l}1238 \mathrm{keV} \text { Line Flux } \\
\left(10^{-4} \mathrm{~cm}^{-2} \mathrm{~s}^{-1}\right)\end{array}$ & $\begin{array}{c}\kappa_{800} \\
\text { Continuum at } 800 \mathrm{keV} \\
\left(10^{-6} \mathrm{~cm}^{-2} \mathrm{~s}^{-1} \mathrm{keV}^{-1}\right)\end{array}$ \\
\hline D86 & $\left\{\begin{array}{l}\mathbf{A} \\
\mathbf{B}\end{array}\right.$ & $\begin{array}{l}<7.8 \\
<6.4\end{array}$ & $\begin{array}{r}<7.9 \\
\ldots\end{array}$ & $\begin{array}{l}\ldots \\
\cdots\end{array}$ \\
\hline D268 & $\left\{\begin{array}{l}\mathbf{A} \\
\mathbf{B}\end{array}\right.$ & $\begin{array}{l}5.3 \pm 6.1 \\
8.8 \pm 5.4\end{array}$ & $\begin{array}{c}13.2 \pm 7.0 \\
\ldots\end{array}$ & $\begin{array}{l}7.3 \pm 3.2 \\
7.1 \pm 3.2\end{array}$ \\
\hline D414 & $\left\{\begin{array}{l}\mathbf{A} \\
\mathbf{B}\end{array}\right.$ & $\begin{array}{l}6.8 \pm 3.2 \\
5.0 \pm 2.8\end{array}$ & $\begin{array}{l}-0.2 \pm 3.5 \\
(<6.7)\end{array}$ & $\begin{array}{l}3.9 \pm 1.7 \\
4.0 \pm 1.7\end{array}$ \\
\hline D771 & $\left\{\begin{array}{l}\mathbf{A} \\
\mathbf{B}\end{array}\right.$ & $\begin{array}{r}<11.1 \\
<7.5\end{array}$ & $\begin{array}{r}<7.3 \\
\ldots\end{array}$ & $\begin{array}{l}\ldots \\
\cdots\end{array}$ \\
\hline
\end{tabular}

NotE.-Upper limits are at the $95 \%$ confidence level.

\section{DISCUSSION}

SN 1987A, a Type II supernova, is associated with the progenitor star Sk $-69^{\circ} 202$ (Sanduleak catalog; decl.: $-69^{\circ}$; No. 202), a blue supergiant of spectral-luminosity class B3 I, with an assumed pre-explosive main-sequence mass in the range 16-20 $M_{\odot}$. The progenitor was a blue supergiant, as opposed to a red star, as would have been anticipated from the existing models of Type II supernovae. In addition, the neutrino burst, in close association with the early optical outburst, provided for the first time the long-anticipated signature of the probable collapse of the $\sim 1.3-1.6 M_{\odot}$ central iron core of the supernova, as had been predicted by theory (see, e.g., Arnett et al. 1989 for a review).

The current interpretation of SN 1987A implies that the core collapse and subsequent rebound generated a shock wave which propagated outward through the star, consistent with the very high emission temperatures inferred from the initial

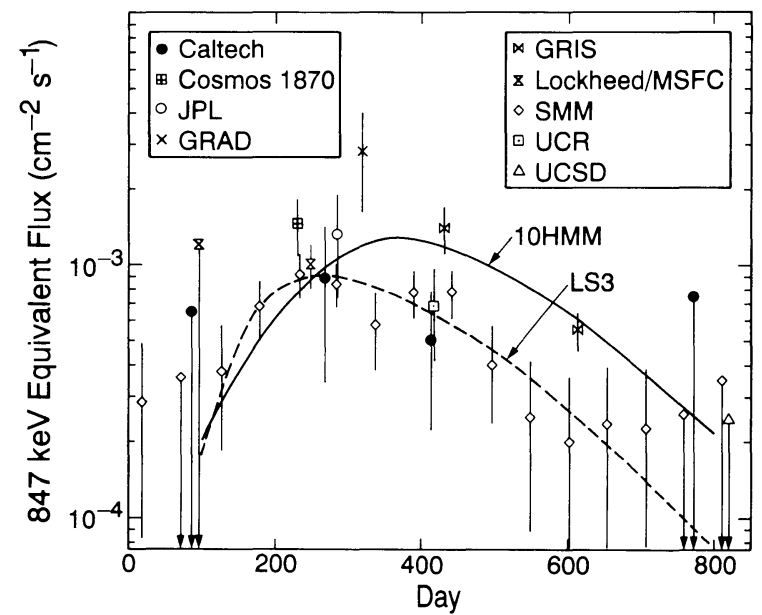

FIG. 6.-Comparison of our line flux measurements with other observations of SN 1987A and with Monte Carlo models. Caltech values are based on the Model B parameter fits of Table 4. Other measurements and models are combinations of 847 and $1238 \mathrm{keV}$ values, combined with an assumed line ratio of 1:0.68, the natural branching ratio for unoccluded ${ }^{56} \mathrm{Co}$. Upper limits are at the $95 \%$ confidence level (Caltech, SMM), $2 \sigma$ level (UCSD), or $3 \sigma$ level (Lockheed/MSFC). The solid and dashed lines are the predicted line flux for Model 10HMM (Pinto \& Woosley 1988a) and for LS3 (Leising \& Share 1990), respectively. The data references are Cosmos 1870 (Efremov et al. 1990); JPL (Mahoney et al. 1988); GRAD (Rester et al. 1989); GRIS (Tueller et al. 1990); Lockheed/MSFC (Sandie et al. 1988a, b); UCR (Ait-Ouamer et al. 1992); and UCSD (Matteson et al. 1990). optical observations. It is now assumed that exterior to the core, the shock wave hydrodynamically heated $\sim 0.08 M_{\odot}$ of material to a temperature in excess of $5 \times 10^{9} \mathrm{~K}$. At this temperature, the material rapidly burned in explosive nucleosynthesis to nuclear statistical equilibrium. This fully processed material would have consisted almost entirely of iron-group elements, primarily ${ }^{56} \mathrm{Ni}$, with sufficient quantities of other isotopes (e.g., ${ }^{57,58} \mathrm{Ni},{ }^{61,62} \mathrm{Zn}$ ) to retain the pre-shock neutronproton ratio (Thielemann, Hashimoto, \& Nomoto 1990). Assuming $\sim 10 \%$ of the initial stellar mass was involved in the initial core collapse and associated rapid nucleosynthesis, the remaining $\sim 90 \%$ of the star would have been thrown explosvely outward by the shock, and would reflect the elemental and isotopic composition developed during the sustained preexplosive burning of the precursor (Arnett et al. 1989).

If the explosion of $\mathrm{SN} 1987 \mathrm{~A}$ had caused the ejecta to expand homologously, or otherwise without mixing, so that spherical symmetry and the original stratified distribution of isotopes was maintained, the ${ }^{56} \mathrm{Ni}$ and its decay products would have remained so deeply buried that detectable levels of line and Compton-scattered continuum flux would not have emerged until about 1 year after the explosion (e.g., Model $10 \mathrm{H}$ of Pinto \& Woosley 1988b). Our initial observations on D86 (Cook et al. 1987), and other measurements at similar times (e.g., Sood et al. 1988), produced only upper limits for both continuum and line emission from SN 1987A. However, evidence for hard X-ray emission (16-28 keV) was reported for SN 1987A from the X-ray astronomy satellite $G I N G A$, as early as D131 (Inoue et al. 1991). In addition, evidence for $24-96 \mathrm{keV}$ emission was reported for D144 from the HEXE experiment on board the Mir-Kvant module, noting that this day corresponds to the initial observation by HEXE of the supernova (Englhauser et al. 1989). Indirect evidence for earlier production of ${ }^{56} \mathrm{Co}$ decay photons and subsequent propagation through the expanding supernova envelope is obtained from the brightening of specific features in the infrared spectrum of SN 1987A for the interval between D76 and D135 (Graham 1988). Evidence for line emission at 847 and $1238 \mathrm{keV}$ first appears at statistically significant levels in data from SMM for the data integration period encompassing D160 to D196 (Leising \& Share 1990). We note that our imaging observation of both continuum and line flux on D268 (Cook et al. 1988c; Palmer 1992), confirmed that the emission was coming from SN 1987A, with little or no contribution from the nearby source LMC X-1.

The early emergence of the hard X-ray and $\gamma$-ray flux indi- 
cates that some fraction of the ${ }^{56} \mathrm{Ni}-{ }^{56} \mathrm{Co}$ had propagated out to optically thin layers of the ejecta. Even if the initial explosion was uniform or symmetric, Rayleigh-Taylor instabilities are expected to force "fingers" or clumps of material outward through the overlying material to lower optical depths (Falk \& Arnett 1973). Further evidence of such large-scale inhomogeneities has been found in the IR spectrum of SN 1987A. The Doppler-broadened profiles of atomic Fe lines indicate structure suggesting that fragments of iron-rich ejecta are traveling at speeds of up to $3000 \mathrm{~km} \mathrm{~s}^{-1}$, significantly greater than the inner edge of the hydrogen envelope $\left(\sim 2100 \mathrm{~km} \mathrm{~s}^{-1}\right)$ (Spyromilio, Meikle, \& Allen 1990). Other IR spectra further suggest that one "bullet," containing at least $3 \%$ of the total $\mathrm{Fe}$ produced, is moving with a line-of-sight velocity of $\sim 3900 \mathrm{~km}$ $\mathrm{s}^{-1}$ in the direction away from us (Haas et al. 1990; Chugai 1992). Evidence obtained from polarimetry data also suggests asymmetry in the explosion (Jeffery 1987; Cropper et al. 1988), as do results associated with the $\gamma$-ray line flux measurements made by the GRIS instrument on days 433 and 613 (Tueller et al. 1990).

Ejecta models developed for SN 1987A after measurements indicated the early appearance of the X-ray and $\gamma$-ray flux have included mixing of heavy core elements in the expanding envelope. These models (e.g., Bussard, Burrows, \& The 1989; Chan \& Lingenfelter 1988; Ebisuzaki \& Shibazaki 1988; Fu \& Arnett 1989; Grebenev \& Sunyaev 1987; Pinto \& Woosley 1988a; Shigeyama \& Nomoto 1990) are consistent with the constraints imposed by the photometric light curve data and the photospheric temperature and velocity histories. These socalled "mixed models" are based on either a spherically symmetric distribution of radioisotopes in the ejecta, or on a fragmented envelope. In early models the mixing was, of necessity, chosen "in an arbitrary fashion" (Pinto \& Woosley 1988a). As additional hard X-ray and $\gamma$-ray measurements were made, the new data further constrained the distribution of the radioisotopes. Leising \& Share (1990) and Sunyaev et al. (1990) have each developed distributions based on their respective flux measurements. The Leising \& Share models, which are based primarily on their line measurements, adequately fit both the observed line flux (see Fig. 6), and the measured continuum above $100 \mathrm{keV}$ as observed by other groups. Sunyaev et al. (1990) have developed a distribution based on measurements of the continuum flux between 45 and $200 \mathrm{keV}$ obtained from Mir-Kvant instruments. As shown in Figure 5, this model agrees with the results of other independent measurements of the continuum emission. In addition, the predictions of $\mathrm{S} 90$ are consistent with the line flux measurements for SN 1987A, being near to or intermediate between the 10HMM and LS3 predictions shown in Figure 6.

From Figures $4 a$ and $4 b$, it is seen that our continuum measurements between $50 \mathrm{keV}$ and $800 \mathrm{keV}$ are significantly lower $(\sim 3.5 \sigma)$ than are predicted by the early, less-constrained $10 \mathrm{HMM}$ model, and are consistent at the $10 \%$ level with the S90 predictions. As seen in Figure 6, existing measurements of the $847 \mathrm{keV}$ line intensity do not strongly favor one model over the other. The measurements made by the GRIS instrument on days 433 and 613 (Tueller et al. 1990) are consistent with the flux predictions of the $10 \mathrm{HMM}$ model, but differ significantly from the $10 \mathrm{HMM}$ predictions with respect to both line widths (2.9 $\sigma$ deviation) and line centroids (4.9 $\sigma$ deviation). In particular, these results may require a nonsymmetric geometry, with both transparent and optically dense regions for the expanding supernova remnant.
From our data for D771, only weak limits can be placed on the quantity of ${ }^{57} \mathrm{Co}$ in the ejecta, and thus the amount of mass-57 material produced (as ${ }^{57} \mathrm{Ni}$ ) in the original explosion. Monte Carlo models indicate that at the time of our D771 observation, ${ }^{57} \mathrm{Co}$ emission would tend to dominate the continuum flux at and below its 122 and $136 \mathrm{keV}$ line energies. Analysis of our continuum data between 77 and $150 \mathrm{keV}$, using as a template the day 800 spectrum in Grebenev \& Sunyaev (1989) with corrections to D771 for isotope decay, shows no evidence for a ${ }^{57} \mathrm{Co}$ enhancement, and allows us to place a $95 \%$ confidence level upper limit on the ratio of mass- $57 /$ mass56 material. We find

$$
\frac{\text { Mass }\left({ }^{57} \mathrm{Ni}+{ }^{57} \mathrm{Co}+{ }^{57} \mathrm{Fe}\right)}{\text { Mass }\left({ }^{56} \mathrm{Ni}+{ }^{56} \mathrm{Co}+{ }^{56} \mathrm{Fe}\right)}<0.24 \text {. }
$$

This upper limit is 10 times the solar system ratio, and can be compared with estimates based on infrared atomic line spectra $(1.5 \pm 0.5$ times the solar system ratio) (Varani et al. 1990), and bolometric light curve data ( $\$ 5$ times the solar system ratio, assuming that the light curve is driven solely by the radioactive decay of ${ }^{57} \mathrm{Co}$ and ${ }^{56} \mathrm{Co}$ ) (Suntzeff et al. 1991, 1992). Also using bolometric observations, Dwek et al. (1992) infer a mass ratio of $4.6 \pm 1.5$ times the solar system value. Other limits have been obtained from hard X-ray and $\gamma$-ray observations made with the HEXE instrument on-board the Mir-Kvant module for the period 1988 September-1989 June (Sunyaev et al. 1990). From these observations, an upper limit of 1.5 times the solar system ratio was obtained. In addition, a balloon-borne hard X-ray observation of SN 1987A on day 1373 by Gunji et al. (1992) has reported upper limits on the total flux emission due to ${ }^{57}$ Co decay. Neglecting any additional contribution to the hard X-ray flux, the resulting upper limit for the mass ratio from these observations is found to be 3.4 times the solar system ratio.

The first determination of the mass $-57 /$ mass- 56 ratio based on direct observation of the hard X-ray flux resulting from the 122 and $136 \mathrm{keV}{ }^{57}$ Co lines from SN 1987A are reported from the Oriented Scintillator Spectrometer Experiment (OSSE) on board the Compton Gamma-Ray Observatory, for the period corresponding to days 1600-1800 (Kurfess et al. 1992). While the ratio obtained by OSSE is to some degree model dependent, a result of $1.5 \pm 0.5$ times the solar system ratio is suggested for low optical-depth models. Further discussion of the OSSE results, including correspondence to theoretical models, appears in Clayton et al. (1992). We note that the quantity of ${ }^{57} \mathrm{Co}$ produced in the supernova provides information on the details of the core explosion, in addition to constraining the neutron excess in the region of the precursor star surrounding the core, allowing constraints to be placed on various models of stellar evolution (Woosley, Pinto, \& Hartmann 1989; Woosley \& Hoffman 1991).

SN 1987A has provided the first opportunity to study in detail the early evolution of the hard X-ray and $\gamma$-ray emission from a Type II supernova. Our observations of the line and continuum emission from SN 1987A, combined with those of other experiments, have placed constraints on models for the nucleosynthesis in the initial explosion, and the subsequent motion and mixing of the ejecta in the expanding remnant. These results are sensitive to the quantity and distribution of nickel-shell material injected into the envelope during the early stages of the expansion.

We acknowledge the important contributions to the devel- 
opment of the GRIP instrument made by W. Althouse, D. Burke, A. Cummings, M. Finger, C. Starr, J. Weger, and the personnel of the Central Engineering Services at the California Institute of Technology. We thank the personnel of the
National Scientific Balloon Facility and the NASA Wallops Flight Facility for their excellent balloon launch support. This work was supported in part by NASA grants NGR 05-002-160 and NAGW-1919.
Ait-Oumer, F., Kerrick, A. D., O'Neill, T. J. Tumer, O. T. Zych, A. D., \& White, S. 1992, ApJ, 386, 715

Althouse, W. E., Cook, W. R., Finger, M. H., Palmer, D. M., Prince, T. A. Schindler, S. M., Starr, C. H., \& Stone, E. C. 1985, Proc. 19th Internat. Cosmic Ray Conf. (La Jolla), 3, 299 1987, Proc. 20th Internat. Cosmic Ray Conf. (Moscow), 1, 84

Arnett, W. D., Bahcall, J. N., Kirshner, R. P., \& Woosley, S. E. 1989, ARA\&A, 27,629

Avni, Y. 1976, ApJ, 210, 642

Bevington, P. R. 1969, Data Reduction and Error Analysis for the Physical Sciences (NY: McGraw-Hill)

Bussard, R. W., Burrows, A., \& The, L. S. 1989, ApJ, 341, 401

Chan, K. W., \& Lingenfelter, R. E. 1988, in AIP Conf. Proc. No. 170, Nuclear Spectroscopy of Astrophysical Sources, ed. N. Gehrels \& G. H. Share (NY: AIP), 110

Chugai, N. 1992, Soviet Astron. Lett., 18(1), 50

Clayton, D. D., Leising, M. D., The, L. S., Johnson, W. N., \& Kurfess, J. D. 1992, ApJ, 400, L141

Cook, W. R., Finger, M. H., Prince, T. A., \& Stone, E. C. 1984, IEEE Trans. Nucl. Sci., NS-31(1), 771

Cook, W. R., Palmer, D. M., Prince, T. A., Schindler, S. M., \& Stone, E. C. 1987, IAU Circ., No. 4400

Cook, W.R., Palmer, D. M., Prince, T. A., Schindler, S. M., Starr, C. H., \& Stone, E. C. 1988 a, IAU Circ. No. 4527 1988b, IAU Circ., No. 4584 1988c, ApJ, 334, L87

Cropper, M., Bailey, J., McCowage, J., Cannon, R. D., Couch, W. J., Walsh, J. R., Strade, J. O., \& Freeman, F. 1988, MNRAS, 231, 695

Dotani, T., et al. 1987, Nature, 330, 230

Dwek, E., Moseley, S. H., Glaccum, W., Graham, J. R., Loewenstein, R. F., Silverberg, R. F., \& Smith, R. K. 1992, ApJ, 389, L21

Ebisuzaki, T., \& Shibazaki, N. 1988, ApJ, 327, L5

Efremov, G. A., Titenkov, A. F., Lupenko, G. V., Nazarova, N. I., Postnikov, I. Y., \& Sukhanov, V. P. 1990, Soviet Astron. Lett., 16(5), 337

Englhauser, J., et al. 1989, Proc. 23d ESLAB Symp. on Two-Topics in X-ray Astronomy, ed. J. Hunt \& B. Battrick (Noordwijk: ESA), 397

Falk, S. W., \& Arnett, W. D. 1973, ApJ, 180, L65

Fenimore, E. E., Klebesadel, R. W., \& Laros, J. G. 1983, Adv. Space Res., 3, 207

Finger, M. H. 1987, Ph.D. thesis, Caltech

Fishman, G. J., Wilson, R. B., Paciesas, W. S., Meegan, C. A., Pendleton, G. N., \& Sandie, W. G. 1990, Adv. Space Res., 10(2), 55

Fu, A., \& Arnett, W. D. 1989, ApJ, 340, 414

Graham, J. R. 1988, ApJ, 335, L53

Grebenev, S. A. \& Sunyaev, R. A. 1987, Soviet Astron. Lett., 13(6), 397

. 1989, in Physics of Neutron Stars, ed. D. A. Varshalovich, A. D. Kaminker, G. G. Pavlov, \& D. G. Yakovlev (NY: Nova Science Publishers), 146

Gungi, T., et al. 1992, ApJ, 397, L83

Haas, M. R., Colgan, S. W. J., Erickson, E. F., Lord, S. D., Burton, M. G., \& Hollenbach, D. J. 1990, ApJ, 360, 257
Heindl, W. A., Cook, W. R., Grunsfeld, J. M., Palmer, D. M., Prince, T. A., Schindler, S. M., \& Stone, E. C. 1993, ApJ, 408, 507

Inoue, H., Hayashida, K., Itoh, M., Kondo, H., Mitsuda, K., Takeshima, T., Yoshida, K., \& Tanaka, Y. 1991, PASJ, 43, 213

Jeffery, D. J. 1987, Nature, 329, 419

Jung, G. V. 1989, ApJ, 338, 972

Kurfess, J. D., et al. 1992, ApJ, 400, L137

Lampton, M., Margon, B., \& Bowyer, S. 1976, ApJ, 208, 177

Leising, M. D., \& Share, G. H. 1990, ApJ, 357, 638 (LS3)

Mahoney, W. A., Varnell, L. S., Jacobson, A. S., Ling, J. C., Radocinski, R. G, $\&$ Wheaton, W. A. 1988, ApJ, 334, L81

Matteson, J., et al. 1990, Proc. 21st Internat. Cosmic Ray Conf. (Adelaide), 2 174

Matz, S. M. Share, G. H., Leising, M. D., Chupp, E. L., Vestrand, W. T. Purcell, W. R. Strickman, M. S. \& Reppin, C. 1988, Nature, 331, 416

Palmer, D. M. 1992, Ph.D. thesis, Caltech

Pinto, P. A., \& Woosley, S. E. 1988a, Nature, 333, 534

. 1988b, ApJ, 329, 820

Rester, A. C., Coldwell, R. L., Dunnam, F. E., Eichhorn, G., Trombka, J. I Starr, R., \& Lasche, G. P. 1989, ApJ, 342, L71

Sandie, W. G., Nakano, G. H., Chase, L. F., Jr., Fishman, G. J., Meegan, C. A., Wilson, R. B., \& Paciesas, W. 1988a, in Supernova 1987A in the Large Magellanic Cloud, ed. M. Kafatos \& A. G. Michalitsianos (Cambridge: Cambridge Univ. Press), 366

Sandie, W. G., Nakano, G. H., Chase, L. F., Jr., Fishman, G. J., Meegan, C. A., Wilson, R. B., Paciesas, W. S., \& Lasche, G. P. 1988b, ApJ, 334, L91

Shelton, I. 1987, IAU Circ., No. 4316

Shigeyama, T., \& Nomoto, K. 1990, ApJ, 360, 242

Sood, R. K., et al. 1988, MNRAS, 234, 73P

Spyromilio, J., Meikle, W. P. S., \& Allen, D. A. 1990, MNRAS, 242, 669

Suntzeff, N. B., Phillips, M. M., Depoy, D. L., Elias, J. H., \& Walker, A. R 1991, AJ, 102(3), 1118

Suntzeff, N. B., Phillips, M. M., Elias, J. H., Depoy, D. L., \& Walker, A. R. 1992, ApJ, 384, L33

Sunyaev, R. A., et al. 1987, Nature, 330, 227

.1990, Soviet Astron. Lett., 16(3), 171 (S90)

Thielemann, F. K., Hashimoto, M., \& Nomoto, K. 1990, ApJ, 349, 222

Tueller, J., Barthelmy, S., Gehrels, N., Teegarden, B. J., Leventhal, M., \& MacCallum, C. J. 1990, ApJ, 351, L41

Ubertini, P., Bazzano, A., Sood, R., Staubert, R., Sumner, T. J., \& Frye, G. 1989, ApJ, 337, L19

Van Allen, J. A., Baker, D. N., Randall, B. A., \& Sentman, D. D. 1974, J Geophys. Res. 79(25), 3559

Varani, G. F., Meikle, W. P. S., Spyromilio, J., \& Allen, D. A. 1990, MNRAS, 245, 570

Woosley, S. E., Axelrod, T. S., \& Weaver, T. A. 1981, Comments Nucl. Part. Phys., 9(6), 185

Woosley, S. E., \& Hoffman, R. D. 1991, ApJ, 368, L31

Woosley, S. E., Pinto, P. A., \& Hartmann, D. 1989, ApJ, 346, 395 